\title{
DEVELOPMENT OF NEXT GENERATION DIGITAL BEAMFORMING SYNTHETIC APERTURE RADAR ARCHITECTURES
}

\author{
Rafael Rincon ${ }^{1}$, Temilola Fatoyinbo ${ }^{1}$, Batuhan Osmanoglu ${ }^{1}$, Seung Kuk Lee ${ }^{1,2}$, K. Jon Ranson ${ }^{1}$, and \\ Guoqing Sun ${ }^{1}$, and Tobias Bollian ${ }^{1,2}$ \\ ${ }^{1}$ NASA Goddard Space Flight Center, Greenbelt, MD \\ ${ }^{2}$ Universities Space Research Association, Columbia, MD
}

\begin{abstract}
Next Generation Digital Beamforming (DBF) Synthetic Aperture Radar (SAR) is a technological area being pursued at the NASA Goddard Space Flight Center (GSFC). Two such systems - DBSAR-2 and EcoSARhave been recently developed and tested. The new instruments employ advanced architectures characterized by multi-mode operation, software defined waveform generation, digital beamforming, and configurable radar parameters. The instruments have been developed to support several disciplines in Earth and Planetary sciences. This paper will describe EcoSAR and DBSAR-2 advanced features and report on the latest SAR processing and calibration efforts.
\end{abstract}

Index Terms-Digital Beamforming, interferometry SAR, InSAR.

\section{INTRODUCTION}

Synthetic Aperture Radar (SAR) systems are indispensable tools in the observation of Earth and Planetary physical properties, as well as in the detection and identification of natural and man-made structures and objects. SAR's unique ability to provide high resolution images in two or three dimensions over large areas, day or night, and under different weather conditions makes it very suitable for remote sensing studies. The applications of SAR span a wide range of scientific, commercial, and military areas, which employ polarimetry, and interferometry techniques [1].

In recent times, emerging digital beamforming (DBF) SAR techniques have been applied to SAR, resulting in enhanced sensor performance and opening a new set of capabilities for future DBF SAR systems. NASA's DBSAR L-band instrument developed in 2008 demonstrated the benefits and capabilities of a DBF SAR [2].

Next Generation Digital Beamforming (DBF) Synthetic Aperture Radar (SAR) can provide even greater enhancing capabilities such as Multiple Input Multiple Output (MIMO) [3][4], radar waveform diversity [5], and single pass interferometry [6]. Most new SAR mission concepts emerging today call for these types of advanced radar systems. These Next Generation Radar systems expand the capabilities of remote sensing radars by enabling new measurements and techniques which overcome several inherent limitations in conventional radar designs.

EcoSAR [7][8] and DBSAR-2 [9] are two advanced airborne radar instrument recently developed at the NASA Goddard Space Flight Center (GSFC). These instruments were designed to provide high quality data in support of several disciplines in Earth and planetary sciences, and to set a path for future spaceborne and airborne SAR missions.

The two instruments build upon the technology path set out by the Digital Beamforming Synthetic Aperture Radar (DBSAR) [2], and incorporate recent state-of-the-art technologies developed under NASA Earth Science Technology Office (ESTO) and Small Business Innovation and Research (SBIR) investments. The instruments are capable of advanced digital radar waveform synthesis and processing; reconfigurable multimode operation; and digital beamforming measurements at high resolution, multiple incidence angle, and full polarimetry.

The EcoSAR and DBSAR-2 measurements are applicable to a number of science study areas ranging from ecosystem structure, surface and sub-surface topography, soil freezethaw, ice sheet composition, glacier depth, and surface water, among many others. In particular, their measurements will provide unique information on vegetation volumes and densities that can be used to map aboveground biomass, forest cover, disturbance from deforestation and degradation, forest recovery, and wetland inundation, helping us quantify carbon release into the atmosphere.

The EcoSAR and DBSAR-2 measurements will also provide an efficient way to detect and document the surface expression of thawing permafrost, helping us quantify the spatial extent of thawing and the accelerating rate of $\mathrm{CO} 2$ and $\mathrm{CH} 4$ gas fluxes to the atmosphere, helping us improve our understanding of the carbon cycle.

Furthermore, the large bandwidth and beamforming operation in these instruments expands their scientific measuring capability by permitting the co-incident reception and processing of reflected signals of opportunity (SoOp) [10]. This capability enables the retrieval of complementary geophysical data, including ocean surface roughness (winds), 
sea height, soil moisture, and ice classification, all relevant to climate change.

\section{ARCHITECTURE}

The EcoSAR and DBSAR-2 radars employs multichannel radar architectures, to perform software defined beam steering on transmit (no moving parts) and digital beamforming on receive. The instruments are characterized by multi-channel operation with software defined waveform generation for each radar transmit channel and dedicated digital receivers for each radar receive channel. Important performance parameters and characteristics achieved with these instruments are listed on Table 1.

Table 1. NEXT GENERATION SAR MAIN PARAMETERS

\begin{tabular}{|l|c|l|}
\hline Frequency & $435 \mathrm{MHz}$ (P-band) & $1.26 \mathrm{GHz}$ (L-band) \\
\hline Bandwidth & $200 \mathrm{MHz}$ & $50 \mathrm{MHZ}$ *, $200 \mathrm{MHz}$ \\
\hline PRF & $1 \mathrm{KHz}$ to $10 \mathrm{kHz}$ & $1 \mathrm{KHz}$ to $10 \mathrm{kHz}$ \\
\hline Pulse Width & $10 \mu \mathrm{s}$ to $30 \mu \mathrm{s}$ & $10 \mu \mathrm{s}$ to $30 \mu \mathrm{s}$ \\
\hline Number of Channels & 32 & 16 \\
\hline Polarization & $\mathrm{HH}, \mathrm{VV}, \mathrm{VH}, \mathrm{HV}$ & $\mathrm{HH}, \mathrm{VV}, \mathrm{VH}, \mathrm{HV}$ \\
\hline $\begin{array}{l}\text { Slant Range } \\
\text { Resolution }\end{array}$ & $0.75 \mathrm{~m}$ & $3 \mathrm{~m}$ \\
\hline $\begin{array}{l}\text { Max. Radiated } \\
\text { Power }\end{array}$ & $40 \mathrm{~W}$ per polarization & $16 \mathrm{~W}$ per polarization \\
\hline $\begin{array}{l}\text { Beam Steering } \\
\text { Range }\end{array}$ & \pm 45 degrees & \pm 45 degrees \\
\hline Antenna Type & Stack-Patch Array & Stack-Patch Array \\
\hline Antenna Size & $2.9 \mathrm{~m} \times 0.8 \mathrm{~m}$ & $1 \mathrm{~m} \times 1 \mathrm{~m}$ \\
\hline
\end{tabular}

*currently limited to $50 \mathrm{MHz}$.

The instrument architectures comprise three main subsystems: the antenna array, the Radar Electronics Unit (REU), and the Radar Digital Unit (RDU), as illustrated in fig. 1.

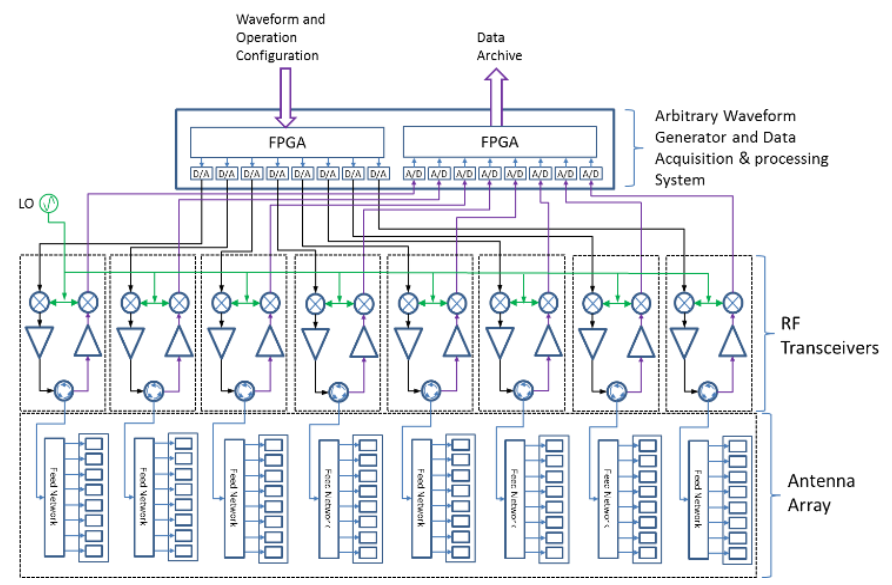

Figure 1. EcoSAR and DBSAR-2 employ multichannel radar architectures comprising three main subsystems: the Radar Digital Unit (RDU), the Radar Electronics Unit (REU), and the antenna. Only 8 channels are shown.
Both instruments employ stacked-patch element antenna arrays (see fig. 2) that permit polarimetric radar operation over large bandwidths $(200 \mathrm{MHz}$ in EcoSAR and up to 500 $\mathrm{MHz}$ in DBSAR-2) and cross-polarization isolation greater than $30 \mathrm{~dB}$. The elements in the array are grouped as subarrays aligned in the flight direction using custom design feed networks.
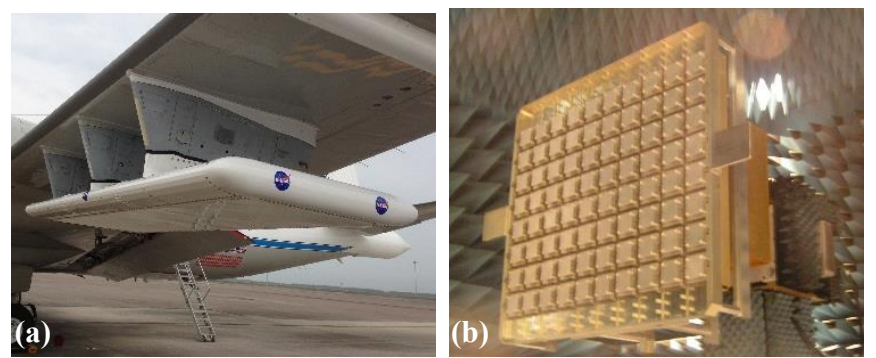

Figure 2. The EcoSAR (a) and DBSAR-2 (b) antennas employ custom designs characterized by polarimetric radar operation over large bandwidths and high cross-polarization isolation.

The Radar Electronics Units (REU) consists of multiple transceiver modules that conditioned transmit and receive signals, provide several calibrations schemes, and support full polarization operation. The $\mathrm{T} / \mathrm{R}$ modules include solid state power amplifier (SSPA), low noise amplifier (LNA), circulator, coupler, filters, control switches and closed loops for robust calibration, dynamic beam control and adaptive waveform generation.

Both instruments employ custom RDUs capable of multichannel arbitrary waveform generation, data acquisition, and onboard processing (see fig. 3). The RDUs, equipped with Vertix 6 FPGAs, digital-to-analog converters (D/As), and analog-to-digital converters (A/Ds), synthesize based band signals with the appropriate phase and amplitude weights for transmit beam steering and side lobe control. Beamforming on receive can be performed on-board, or processed off-line by coherently capturing the raw complex data. The RDUs are also responsible for radar timing, and data transfers.

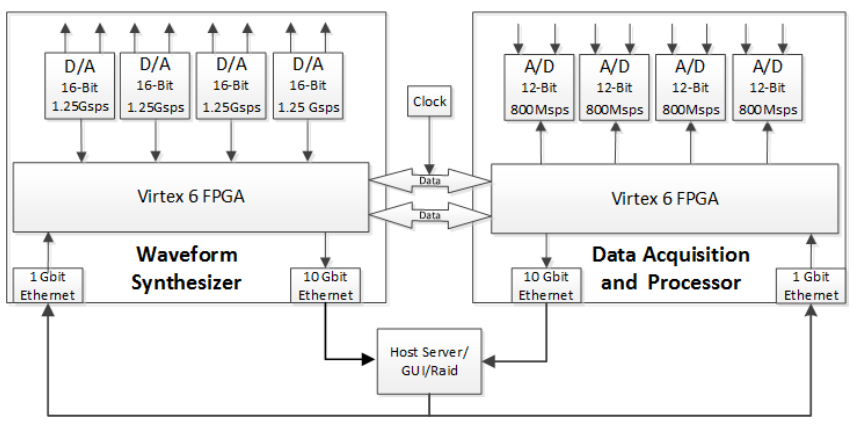

Figure 3. EcoSAR and DBSAR-2 employ custom Radar Digital Units (RDUs) responsible for multi-channel waveform generation, data acquisition and onboard processing. 
The RDUs include host linux servers that run a custom Graphical Unit Interface (GUI) (see fig. 3) running on Linux based servers which performs real-time configuration and housekeeping of the instruments, as well as data monitoring and archiving.

The antenna, REU and RDU arrangement (fig. 1) allows for independent amplitude and phase control at each subarray on transmit, and amplitude and phase measurements between any pair of subarrays on receive, thus enabling across-track scanning. The architecture allows considerable measurement flexibility including the generation of customized transmit and receive beams, imaging both sides of the flight track (simultaneously or time interleaved), post processing synthesis of multiple beams, variable incidence angle, swath width and ground resolution.

\section{INSTRUMENT STATUS}

EcoSAR's first flights were conducted over areas of Andros Island in the Bahamas, and the La Selva and Corcovados National Parks in Costa Rica in the spring 2014. The instrument flew onboard a NOAA P3 aircraft for approximately 19 hours for data collections and transit to the sites. Field measurements over these areas were also conducted during the same month.

During the campaign, EcoSAR collected full pol interferometric SAR data at $50 \mathrm{MHz}, 120 \mathrm{MHz}$, and $200 \mathrm{MHz}$ bandwidths in standard and Ping Pong modes. SAR and InSAR image analysis is currently under way. The results from the analysis as well as system performance results will be included in the full paper and presented at the conference.

DBSAR-2 was fully characterized at NASA Goddard's Electromagnetic Anechoic Chamber (GEMAC). The chamber measurements were used to generate steering vector coefficiecients, and transmit and receive beam patterns were measured at various look angles and amplitude tapers.

Two-way measurements in both polarizations and cross pols were also performed in the anechoic chamber using an optical delay line system. The measurements allowed us to test the full operational functionality of the system (Tx and $\mathrm{Rx}$ beam steering, radar timing, two way gain and dynamic range, and radar impulse response).

DBSAR-2 is scheduled to perform first flight tests in the summer 2016. Although, DBSAR-2 is compact to allow installation on a range of aircrafts, our next step is to integrate it and fly it on the NASA's P3 and C-130 aircrafts. The antenna radome design, originally design for the $\mathrm{P} 3$, can also be flown on the C-130 through the use of a custom aerodynamic fairing.

\section{CONCLUDING REMARKS}

The EcoSAR and DBSAR-2 are the latest advances in digital beamforming SAR for Earth science applications. These developments seek to demonstrate the Next Generation Digital beamforming SAR. Both instruments have been completed, integrated and calibrated in the laboratory and in the anechoic chamber. EcoSAR's first flights were conducted the spring 2014. Data analysis and performance assessment is under way. DBSAR-2's first test flights are scheduled in summer of 2016.

The EcoSAR and DBSAR-2 technology will help established the Next Generation DBSAR capability as a science instrument while setting a path future Earth science and planetary exploration SAR missions.

\section{REFERENCES}

[1] Henderson, F. M.; Lewis, A. J. Manual of Remote Sensing, Volume 2: Principles and Applications of Imaging Radar. John Wiley \& Sons, New York, $3^{\text {rd }}$ edition, 1998.

[2] Rincon, R. F.; Vega, M. A.; Buenfil, M.; Geist, A.; Hilliard, L.; Racette, P.; 2011A, "NASA's L-Band Digital Beamforming Synthetic Aperture Radar," IEEE Trans. Geosci. Remote Sens.,, vol.49, no.10, pp.3622-3628, Oct. 2011 doi: 10.1109/TGRS.2011.2157971.

[3] Krieger, G; "MIMO-SAR: Opportunities and Pitfalls", IEEE Trans. Geosci. Remote Sens., vol.52, no.5, pp.2628-2645, Jun. 2013.

[4] W.-Q. Wang, "Space-time coding MIMO-OFDM SAR for highresolution imaging," IEEE Trans. Geosci. Remote Sens., vol. 49, no. 8, pp. 3094-3104, Aug. 2011.

[5] Krieger, G; Gerbert, N.; and Moreira, A.; "Multidimensional Waveform Encoding: A New Digital Beamforming Technique for Synthetic Aperture Radar Remote Sensing", IEEE Trans. Geosci. Remote Sens.,, vol.46, no.1, pp.31-36, Jan. 2008.

[6] Rincon, R.; Fatoyinbo, T.; SeungKuk Lee; Osmanoglu, B.; Ranson, J.; Guoqing Sun; "Digital beamforming synthetic aperture radar (DBSAR): Single-pass interferometry for forest structure estimation". Geoscience and Remote Sensing Symposium (IGARSS), 2015.

[7] Fatoyinbo T., R. Rincon, G. Sun, K. J. Ranson, 2011, EcoSAR: A P-band Digital Beamforming Polarimetric Interferemetric SAR Instrument to Measure Ecosystem Structure and Biomass, Proc. IEEE Int. Geosci. Rem. Sens. Symp., July 25-29, 2011, Vancouver, Canada.

[8] Rincon R., Fatoyinbo T., Ranson K., Osmanoglu B., Lee S., Ranson K. J., Sun G, Perrine M., and Du Toit C.; EcoSAR: PBand Digital Beamforming Polarimetric And Single Pass Interferometric SAR. Radar Conference, 2015 IEEE. Radar Conference, 2015 IEEE; May 2015.

[9] Rincon, R.; Fatoyinbo, T.; Osmanoglu, B.; Seung-Kuk Lee; Ranson, K.J.; Marrero, V.; Yeary, M; "Next generation Digital Beamforming Synthetic Aperture Radar (DBSAR-2)". Geoscience and Remote Sensing Symposium (IGARSS), 2015.

[10] Shah, R.; Garrison, J.L.; and Grant, M.S., "Demonstration of Bi-static Radar for Ocean Remote Sensing Using Communication Satellite Signals", IEEE Geoscience and Remote Sensing Letters, vol. 9 , no. 4, May 2012. 